\title{
On the Sensitivity of Zonal-Index Persistence to Friction
}

\author{
PABLO ZURITA-GOTOR \\ Departamento de Geofísica y Meteorología, Universidad Complutense, and \\ Instituto de Geociencia, Madrid, Spain
}

(Manuscript received 17 March 2014, in final form 10 June 2014)

\begin{abstract}
Friction is an important parameter in atmospheric modeling that may affect internal variability in a number of ways. It directly damps the annular-mode variability, but it also helps to maintain it through baroclinic feedbacks. Also, by determining the mean strength and position of the midlatitude jet, friction affects the internal dynamics that drive this variability. This work investigates the relevance of all of these factors for the sensitivity of the persistence of annular variability to changes in the zonal-mean friction using an idealized quasigeostrophic two-layer model. This model produces realistic variability, yet it is so simple that one can cleanly separate the different effects. It is found that the sensitivity of persistence to friction is dominated by the direct damping effect, while changes in the eddy momentum forcing and in the mean jet are not as important.

As in more complex models, the persistence of the jet anomalies in this model decreases at all lags with increasing friction, but the long-time decorrelation decay rate of these anomalies is remarkably insensitive to friction. Although this implies that the eddy feedback must increase with friction to maintain the anomalies against the enhanced damping, it is shown that this is not due to baroclinic effects. A model that assumes that the eddy forcing does not change with friction can reproduce reasonably well the numerical results. The crucial factor that determines the model's sensitivity to friction is the spectral structure of the eddy momentum forcing.
\end{abstract}

\section{Introduction}

Surface friction is one of the main dissipative terms in Earth's atmosphere, providing the primary sink for the eddy kinetic energy generated by baroclinic instability in the midlatitude troposphere (Peixoto and Oort 1992). However, the importance of surface friction for the general circulation is not limited to this role: through its effect on the velocity structure of the mean flow, friction also affects the efficiency of energy conversions (James 1987) and how waves propagate (Barnes and Garfinkel 2012). This may have nontrivial effects on the mean state, affecting eddy kinetic energy in unexpected ways (James 1987) and the jet position as well as its strength (Robinson 1997; Chen et al. 2007).

This work is concerned with the sensitivity of the internal annular variability of the atmosphere to changes

Corresponding author address: Pablo Zurita-Gotor, Facultad de Ciencias Físicas, Departamento de Geofísica y Meteorología, Universidad Complutense de Madrid, Avenida Complutense s/n, Madrid 28040, Spain.

E-mail:pzurita@alum.mit.edu in surface friction. When a velocity norm is used, this variability may be described in terms of the latitudinal excursions of the eddy-driven jet and is governed by the simple equation (Lorenz and Hartmann 2001)

$$
\frac{\partial z}{\partial t} \approx m-\frac{z}{\tau_{Z}},
$$

where $z$ is the zonal index, a measure of the jet position; $m$ is the forcing by the eddy momentum flux; and $\tau_{Z}$ is a frictional time scale (see section 2 for more details). As a damping term, the first effect of friction is to reduce the variance of the variability. Additionally, it may affect its persistence. For instance, when $m$ is white, $z$ decorrelates with $\tau_{Z}$.

Lorenz and Hartmann (2001) showed that in fact the $m$ spectrum is not white but displays enhanced power at low frequencies. They attributed this low-frequency power to the organization of the synoptic eddies by the mean flow, which they modeled using a linear feedback model: $m=b z+\tilde{m}$, where $\tilde{m}$ is that part of the eddy forcing independent of $z$ and $b$ is a feedback coefficient. Because this coefficient is positive, the coupling 
between the mean-flow and eddy forcing anomalies amplifies the low-frequency variability and enhances the persistence of $z$. Substituting $m=b z+\tilde{m}$ in the $z$ tendency equation, it follows that $z$ decays at long lags (after the memory of $\tilde{m}$ is lost) as a red-noise process with a reduced damping scale: $a=\left(1 / \tau_{Z}\right)-b$. One can thus estimate the eddy feedback coefficient $b$ from the long-lag decay rate of the zonal-index autocorrelation $a$, as described in detail in the next section.

Thus, the sensitivity of zonal-index persistence on friction may be quite different depending on how $b$ changes. This is still an open question, as the dynamics of the positive feedback remains a topic of active investigation (Barnes and Thompson 2014; Zurita-Gotor et al. 2014; Simpson et al. 2013). Barotropic theories attribute the dependence of $m$ on $z$ to changes in uppertroposphere wave propagation associated with changes in the upper-level jet (Chen and Zurita-Gotor 2008; Lorenz 2014), whereas baroclinic theories rely on changes in eddy generation resulting from the accompanying changes in the baroclinicity (Blanco-Fuentes and ZuritaGotor 2011). Whether by limiting the upper-level jet acceleration or by generating anomalous baroclinicity (Robinson 2000), it seems plausible that friction might play a role in both scenarios.

Besides the barotropic and baroclinic effects of friction on the anomalous jet, friction may also affect lowfrequency variability through its impact on the mean state (Chen and Plumb 2009). When friction is reduced the jet strengthens and narrows, and on the sphere it also moves poleward (Robinson 1997). This affects the interaction between the eddies and the Hadley cell and the characteristics of wave propagation (Barnes et al. 2010), and several studies have found a relation between the latitude of the mean jet and the persistence of the internal variability (Kidston and Gerber 2010; Arakelian and Codron 2012). Even if the jet does not shift, its barotropic and/or baroclinic structure might be important for the internal dynamics that drive the lowfrequency variability.

To understand the sensitivity of zonal-index persistence to friction, it is necessary to separate all these effects, so that their relative importance can be assessed. Our work is strongly motivated by the study of Chen and Plumb (2009), who devised a method to change the frictional time scale without letting the mean flow vary. With this simplification, the authors found that changes in the eddy feedback very nearly compensated the changes in the frictional damping rate, so that the rate of decorrelation of the low-frequency mean-flow anomalies changed very little with friction. Chen and Plumb (2009) speculated that this could be due to an enhanced baroclinic eddy feedback with stronger friction, though it is unclear why the dynamics would seek such a perfect compensation.

We have devised a formulation that allows us to change the barotropic and baroclinic effects of friction independently in the two-layer quasigeostrophic model, and hence to test the impact of baroclinic feedback changes for the model's sensitivity to friction. We already described in detail in a previous study the internal variability of this model (Zurita-Gotor et al. 2014, hereinafter Z14), which is strikingly realistic despite all of its simplifications. In particular, the eddy forcing spectrum in this model exhibits a low-frequency peak similar to the observations of Lorenz and Hartmann (2001) even though our model has no time-dependent forcing or planetary waves.

This paper is organized as follows. Section 2 briefly introduces the model and describes its internal variability. Section 3 investigates the sensitivity of persistence in this model to friction, isolating its different effects. It is shown that although the results are consistent with Chen and Plumb (2009), baroclinic feedbacks only play a small role. Section 4 rationalizes our results and shows that one can reproduce the essential behavior with fixed eddy forcing. We close with some concluding remarks in section 5 .

\section{The two-layer model and its annular variability}

We use for this study a standard two-layer quasigeostrophic model on a beta channel. The model is forced by Newtonian relaxation to an unstable "radiative equilibrium" profile defined by the hyperbolic secant thermal wind jet: $-\partial \psi_{R} / \partial y=40 \mathrm{~ms}^{-1} \times \operatorname{sech}^{2}(y / 2500 \mathrm{~km})$, with time scale $\tau_{D}=20$ days. The model is damped by Rayleigh friction with time scale $\tau_{F}=3$ days in the lower layer only, and biharmonic diffusion with $\nu=5 \times 10^{15} \mathrm{~m}^{4} \mathrm{~s}^{-1}$ is used to dissipate the direct enstrophy cascade. The model uses a deformation radius $\lambda=700 \mathrm{~km}$ (based on the layer depth) and a typical midlatitude $\beta=1.6 \times 10^{-11} \mathrm{~m}^{-1} \mathrm{~s}^{-1}$. The model and control parameters are the same as in $\mathrm{Z} 14$, to which the reader is referred for additional details.

Figure 1a describes the climatological zonal wind (blue) and its leading mode of variability (red), expressed as the regression of the barotropic zonal-mean zonal wind on the standardized principal component time series for its leading EOF. This leading EOF represents a shift of the jet about its mean position and the associated principal component is called the zonal index. Using the momentum equation, the evolution of the zonal index is governed by the simple equation (Lorenz and Hartmann 2001)

$$
\frac{\partial z}{\partial t}=m-\text { Fric }
$$


a) Zonal wind and leading EOF

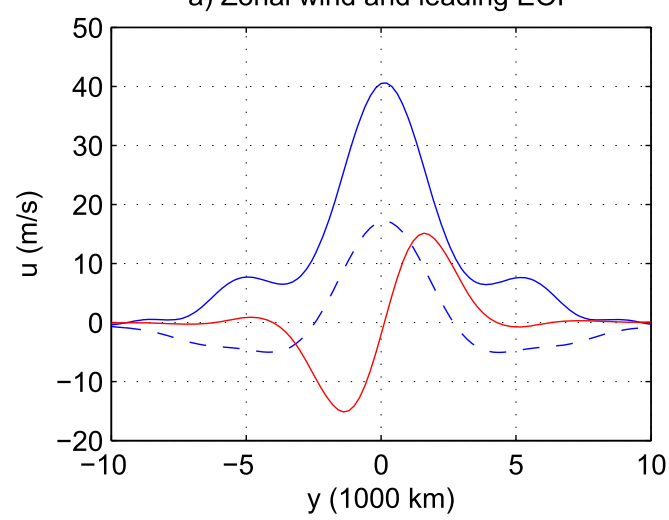

c) $\partial \log c_{z z} / \partial \tau$ (days $\left.^{-1}\right)$

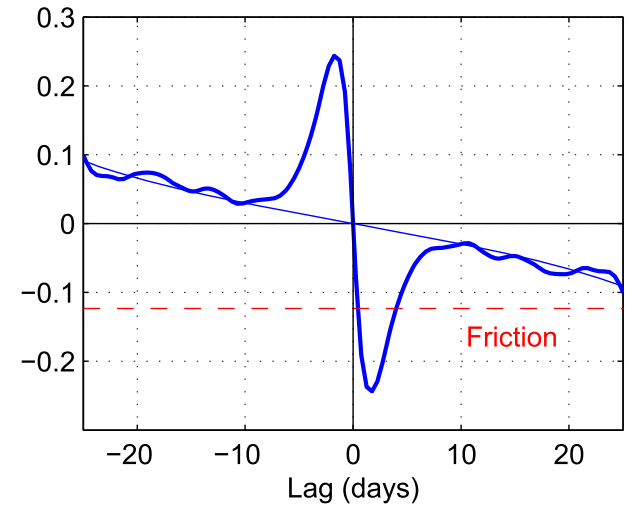

b) Zonal index autocorrelation

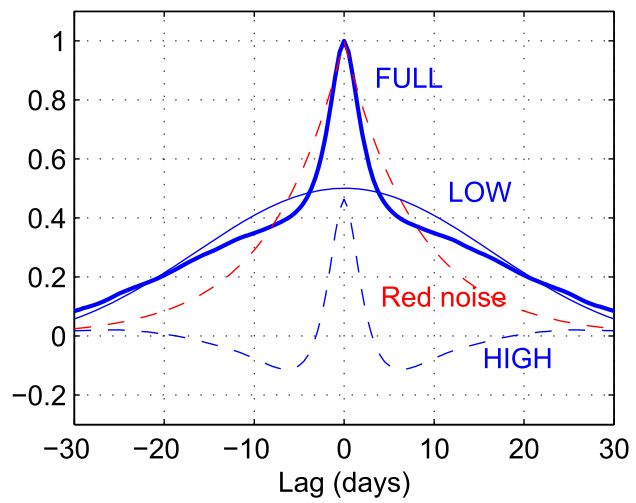

d) Power spectral density (normalized)

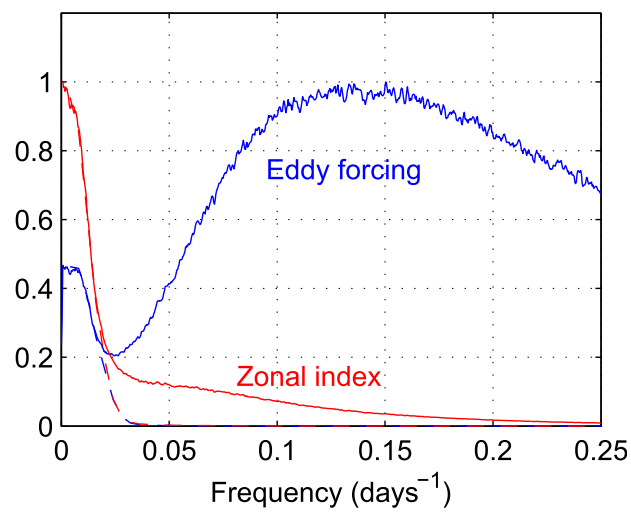

FIG. 1. (a) Climatological zonal wind (blue; lower layer dashed) and barotropic zonal-mean zonal wind associated with one standard deviation of the principal component time series for its leading EOF (red). (b) Zonal-index autocorrelation (thick solid blue), and contributions by the low (thin solid blue) or high (thin dashed blue) frequency components of the eddy forcing spectrum. The autocorrelation function for a red-noise process with the same damping is also shown (dashed red). (c) Logarithmic decay rate of the autocorrelation function for the zonal index (thick blue) and its low-frequency component (thin blue), compared to the frictional time scale (dashed red). (d) Power spectra of zonal index (red) and its eddy forcing (blue), and the filtered forcings input to the offline model in (b).

where $z=\int\left(\left[u_{1}\right]+\left[u_{2}\right]\right) \cdot \mathbf{e}(y) d y$ is the zonal index, $m=-\int \partial\left(\left[u_{1}^{*} v_{1}^{*}\right]+\left[u_{2}^{*} v_{2}^{*}\right]\right) / \partial y \cdot \mathbf{e} d y$ is the eddy momentum forcing, and Fric $=\left(1 / \tau_{F}\right) \int\left[u_{2}\right] \cdot \mathbf{e}(y) d y$ is the frictional damping. Square brackets denote timedependent zonal averages and asterisks denote deviations from these averages; subscripts 1 and 2 refer to the upper and lower layers, respectively; and $\mathbf{e}(y)$ is the normalized EOF shown in Fig. 1a.

Z14 show that the temporal and spectral properties of the zonal index can be well reproduced by integrating Eq. (1) directly using the simulated $m$ time series and approximating the frictional term as Fric $\approx-z / \tau_{z}$. The frictional time scale on the zonal index is expressed as $\tau_{Z}=\alpha \tau_{F}$, where $\alpha$ is a parameter related to the vertical structure of the modes. For our control run, the best fit is provided by $\alpha=2.7$ (see Z14 for details), but this parameter increases (decreases) slightly with stronger (weaker) friction, as the modes become more (less) top heavy. We will refer to this simple integration procedure as the "offline model." What makes this model so useful is that it allows us to disentangle changes in the persistence of $z$ due to changes in $m$ from those due to changes in $\tau_{Z}$ (the "direct damping effect").

Figure $1 \mathrm{~b}$ compares the simulated zonal-index autocorrelation in our model $c_{z z}(\tau)=\left\langle z^{\prime}(t+\tau), z^{\prime}(t)\right\rangle /\left\langle z^{\prime}(t)^{2}\right\rangle$ with the expected autocorrelation function for a firstorder autoregressive (AR1) model with the same damping time scale: $c_{z z}^{\text {red }}=\exp \left(-|\tau| / \tau_{Z}\right)$. The differences between these two curves are due to the eddy memory (Lorenz and Hartmann 2001). At short lags, $m$ is negatively autocorrelated and decreases zonal-index persistence. At long lags, the anomalous $m$ tends to reinforce the $z$ anomalies (a positive eddy feedback) and the zonal-index autocorrelation decays more slowly than 
friction. As discussed by Z14, both the short-lag negative eddy forcing and the long-lag positive eddy feedback are stronger in this run than in observations, producing a noticeable shoulder in the zonal-index autocorrelation.

Z14 proposes estimating the positive eddy feedback as the difference between the logarithmic decay rate of the zonal-index autocorrelation at long lags, $a=-\partial \log c_{z z} /$ $\partial \tau$, and the frictional damping rate $\tau_{Z}^{-1}$. In the simplest linear feedback model (Lorenz and Hartmann 2001), $m=b z+\tilde{m}$ with $b$ constant and $\tilde{m}$ and $z$ uncorrelated at long lags; then, as discussed in the introduction, the longlag autocorrelation decays exponentially as in the AR1 model, albeit with a reduced damping rate $a=1 / \tau_{Z}-b$. However, it is apparent in Fig. 1c that this simple model does not work too well in our simulations, in which $\partial \log c_{z z} / \partial \tau$ is not constant at long lags but decreases monotonically with $\tau$, to a good approximation linearly. This behavior implies that the long-lag autocorrelation is better approximated by a Gaussian function, $c_{z z} \sim \exp \left[-(1 / 2) a^{2} \tau^{2}\right]$, than by $\exp (-a|\tau|)$. Since the autocovariance and the power spectrum form a Fourier pair, this suggests that the low-frequency zonal-index spectrum is better characterized by a Gaussian than a Lorentzian profile. Using an analogy from line shape spectroscopy, a possible interpretation of these results is that $b$ should be regarded as a distribution rather than a constant value.

Figure 1d shows the normalized power spectra for the zonal index $p_{z}(\omega)=Z Z *$ and the eddy forcing $p_{m}(\omega)=$ $M M^{*}$ (we use capital letters to denote Fourier transforms and asterisks for complex conjugates), constructed averaging 3600 realizations over more than 15000 years of simulations. The eddy forcing spectrum consists of two peaks: a sharp peak centered at near-zero frequencies and a much broader peak centered at periods between 5 and 10 days, associated with the synoptic eddy spectrum. Although the low-frequency peak captures a very small fraction of the total $m$ variance, this peak is certainly important for $z$ [note that from Eq. (1) both spectra are related by $\left.p_{z}=\left(\omega^{2}+\tau_{Z}^{-2}\right)^{-1} p_{m}\right]$. As discussed by Z14, the zonal-index time series is given by the sum of the responses to both spectral peaks in the eddy forcing spectrum. We can separate the contributions from each spectral peak to the total zonal-index autocorrelation, forcing the offline model with the highor low-pass-filtered $m$ time series. Figure 1 b shows that, while the high-frequency part of the $m$ spectrum is responsible for the faster-than-frictional zonal-index decay at short lags, the response to this forcing dies out (because this peak is much broader) and the autocorrelation is dominated by the response to the lowfrequency peak at long lags. This response has a broad Gaussian shape and its logarithmic decay rate increases linearly with lag (thin blue line in Fig. 1c), as noted above.

If we model the low-pass-filtered autocorrelation as a Gaussian $c_{z z}^{\text {low }} \sim \exp \left[-(1 / 2) a^{2} \tau^{2}\right]$, then the lowfrequency peak in the $z$ spectrum should also have a Gaussian shape: $p_{z} \sim \exp \left(-\omega^{2} / \delta_{\omega}^{2}\right)$ with $\delta_{\omega}=a$ (up to a normalization factor). Although zonal-index persistence will be sensitive to the relative amplitude of the low- and high-frequency peaks, the long-lag decay rate of the autocorrelation-and hence the eddy feedbackshould only depend on $\delta_{\omega}(=a)$. An example is provided by the sensitivity of persistence to diffusion in our model, discussed in Z14. As diffusion increases, the highfrequency part of the eddy forcing spectrum weakens and the zonal index becomes more persistent. However, the low-frequency spectrum and the long-lag logarithmic decay rate of the zonal-index autocorrelation, which emerge once the response to the high-frequency spectrum has died out, remain unchanged.

It is actually easier to estimate $a$ using the autocorrelation function than to estimate $\delta_{\omega}$ from the power spectrum, which requires good spectral resolution at low frequency. Since the temporal and spectral structures described above are very robust (in every simulation that we have examined, varying virtually all model parameters, the logarithmic decay rate of the zonal-index autocorrelation displays a similar structure to Fig. 1c), we can estimate the eddy feedback as follows. First, we low-pass filter the eddy forcing time series using a cutoff of 40 days. Then, we calculate the zonal-index response to this forcing using the offline model and compute the logarithmic decay rate of its autocorrelation function. Finally, we take the value of this decay rate (roughly linear in $\tau$ ) at lag $\tau=10$ days as our estimate of the longlag decay rate, and its difference from the damping time scale as an estimate of the eddy positive feedback. As we shall see, this gives similar but more robust results to the method proposed by Lorenz and Hartmann (2001) in our model.

\section{Model sensitivity to friction}

As discussed in the introduction, friction can affect the persistence of internal zonal-mean variability in multiple ways. In this section we discuss the results of some targeted sensitivity experiments designed to test the relevance of the different mechanisms.

Figure 2a shows how the zonal-index autocorrelation changes when the frictional time scale is varied from a third of its control value to 3 times that value. Only the zonal-mean friction is changed in these experiments, while the friction acting on the eddies is kept constant at its control value $\tau_{F}=3$ days (friction on the mean and 
a) Zonal index autocorrelation

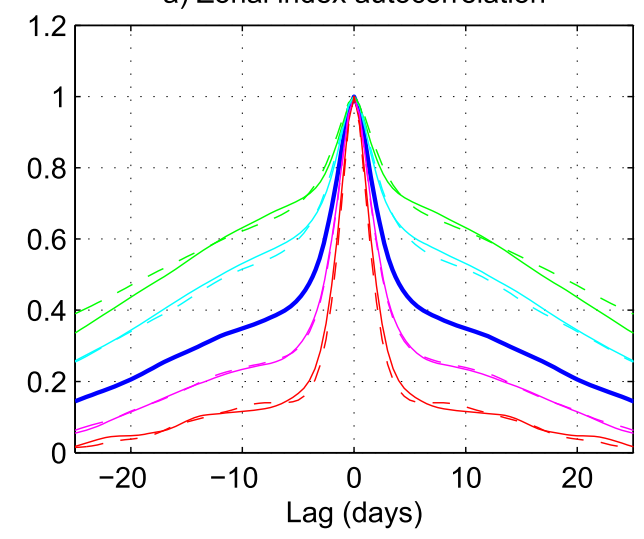

c) $\partial \log c_{z z} / \partial \tau$ (days $\left.^{-1}\right)$

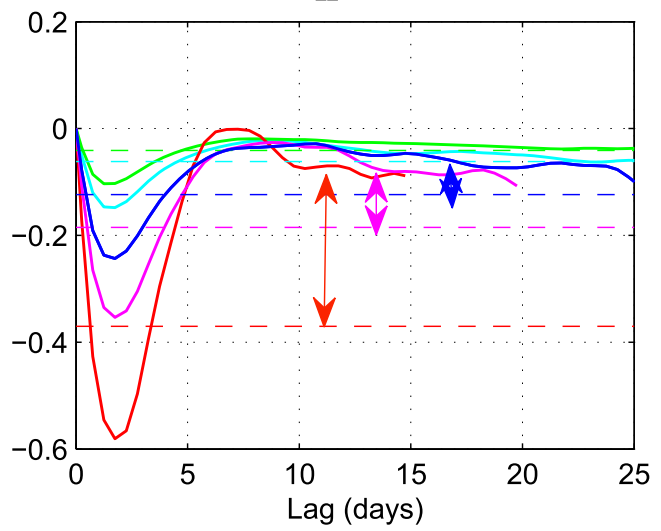

b) Lower layer wind $(\mathrm{m} / \mathrm{s})$

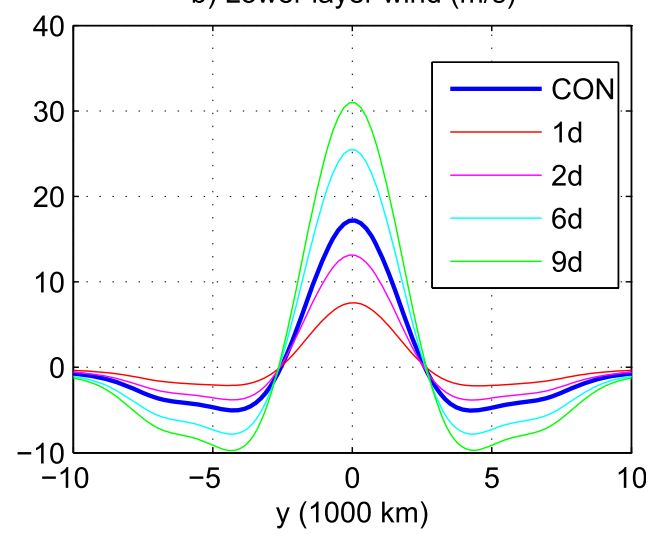

d) Eddy feedback (days ${ }^{-1}$ )

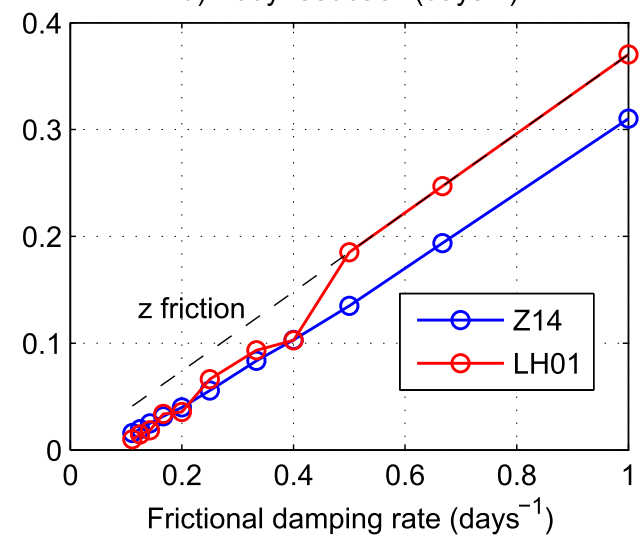

FIG. 2. (a) Sensitivity of zonal-index autocorrelation on the zonal-mean frictional time scale [red: 1 day, magenta: 2 days, blue: 3 days (control), cyan: 6 days, and green: 9 days]. The dashed lines show the same when changing the frictional time scale in the direction of the leading EOF alone (see text for details). (b) As in (a), but for lower-layer wind. (c) Logarithmic decay rate of zonal-index autocorrelation for the same simulations (solid), compared to the frictional time scale in each case (dashed). Their long-lag differences (double arrows) provide a measure of the eddy positive feedback. (d) Sensitivity of eddy feedback to the frictional time scale estimated as in (c) (blue) or using the method of Lorenz and Hartmann (red), compared to the effective friction acting on the zonal index, $\tau_{Z}^{-1} \approx\left(2.7 \tau_{F}\right)^{-1}$ (dashed black).

the eddies tend to have opposite effects, but the former is usually dominant in weakly dissipative regimes; e.g., Chen et al. 2007). Changes in the mean friction have a profound impact on the mean state for this model (Zurita-Gotor 2007): as friction is reduced, a strong barotropic jet with large meridional curvature develops (Fig. 2b) and the eddy energy level decreases through the barotropic governor mechanism (James 1987).

Changes in friction also affect the persistence of annular variability, with the zonal index becoming more persistent at all lags as friction is reduced (Fig. 2a). Figure $2 \mathrm{c}$ shows that this is mostly due to the more rapid short-lag decay of the zonal-index autocorrelation with increasing friction. In contrast, the long-lag decay rate of the autocorrelation only weakens slightly as friction is changed by roughly an order of magnitude. The latter implies that the positive eddy feedback must increase with friction, as indicated by the double arrows in Fig. 2c. This is confirmed by Fig. 2d, which shows the sensitivity to friction of $b$, estimated as explained in section 2. The results are very similar when the eddy feedback is estimated using the method proposed by Lorenz and Hartmann (2001), which entails finding the value of $b$, which minimizes the lagged correlation between $m$ and $z$ for moderate and large $z$ leads after the feedback is removed. However, that method fails with large friction (the lagged correlation has no local minimum in those cases and the optimization procedure simply gives the interval limit, the frictional time scale).

Although the reported sensitivity of zonal-index persistence to friction is consistent with stronger damping of the variability with large friction, it is not clear whether 
changes in the mean state (cf. Fig. 2b) might also play a role. To address this question, we have performed a series of simulations in which the mean state is kept fixed as friction is varied, following Chen and Plumb (2009). This is done by changing the frictional time scale in the direction of the leading EOF (the jet shift) alone. Specifically, we calculate the lower-layer zonal-mean friction using the expression

$$
\left[F_{2}\right](y, t)=-\frac{U_{\mathrm{EOF}}}{\tau_{\mathrm{EOF}}}-\frac{\left[u_{2}\right]-U_{\mathrm{EOF}}}{\tau_{F}},
$$

where $u_{2}$ is the lower layer wind and $U_{\mathrm{EOF}}(y)=$ $\left(\left[u_{2}\right] \cdot \mathbf{e}\right) \mathbf{e}(y)$ is its component in the direction of the leading EOF $\mathbf{e}(y)$. Since by the symmetry of the model $\left[\bar{u}_{2}\right]$ (the overbar indicates a time mean) is symmetric about midchannel, $\bar{U}_{\mathrm{EOF}}$, which is antisymmetric, must vanish and hence $\left[\bar{F}_{2}\right]$ and the model's climatology are insensitive to changes in $\tau_{\mathrm{EOF}}$ as long as $\tau_{\mathrm{EOF}}$ is finite. Additionally, it was found empirically that the meridional structure of $\mathbf{e}(y)$ is slightly sensitive to changes in $\tau_{\mathrm{EOF}}$, in contrast to the variance explained by this mode (the jet shift ceases to be the dominant mode with sufficiently strong friction). Thus, the results are unchanged when $U_{\mathrm{EOF}}$ is computed using the EOF of the perturbed friction run instead of that of the control run, and it is not necessary to devise an iterative procedure changing $\mathbf{e}(y)$ in each step. Similar results to those described below are obtained when the mean state is kept fixed by the addition of a constant torque as in Chen and Plumb (2009), but that method diverges for large changes in friction. The relation between both methods is discussed in the appendix.

Figure 3 shows that $\tau_{\mathrm{EOF}}$ has a large impact on the persistence of annular variability. Compared to the control simulation (Fig. 3a), the meridional excursions of the jet become larger and more persistent when the EOF friction is reduced (Fig. 3b). Since the mean state is now the same for both simulations (cf. the green and blue lines in Fig. 3d), this must be due to the reduced damping of the variability with weaker friction. One can even take the limit $\tau_{\mathrm{EOF}} \rightarrow \infty$ (Fig. 3c, note the different horizontal scale compared to the previous panels), in which case the meridional excursions of the jet proceed undamped. This affects the fundamental character of the annular variability: whereas in the previous two cases the probability distribution function (PDF) for the principal component of the jet shift was Gaussian, in the absence of friction the distribution becomes bimodal (Fig. 3e) and there are two preferred equilibrium states, with jets pushed to one or the other margin of the baroclinic zone.

The zonal-index autocorrelations for the simulations' varying $\tau_{\mathrm{EOF}}$ are very similar to those described above changing the full friction (cf. the solid and dashed lines in Fig. 2a), which suggests that changes in the mean flow are relatively unimportant for our model's sensitivity to friction (the results may be different on the sphere). What seems harder to understand is why the eddy feedback should increase so efficiently with friction as to leave the zonal-index logarithmic decay rate nearly unchanged at long lags (cf. Figs. 2c,d). The same was found by Chen and Plumb (2009), who speculated that this might be due to an enhanced baroclinic eddy feedback with increased friction (Robinson 2000). To test this idea, we have devised a formulation that allows us to distinguish between the barotropic and baroclinic effects of friction. We replace Eq. (2) with the following:

$$
\begin{aligned}
& {\left[F_{1}\right]=-\left(\frac{1}{2 \tau_{\mathrm{BT}}}-\frac{1}{2 \tau_{\mathrm{BC}}}\right) U_{\mathrm{EOF}}} \\
& {\left[F_{2}\right]=-\left(\frac{1}{2 \tau_{\mathrm{BT}}}+\frac{1}{2 \tau_{\mathrm{BC}}}\right) U_{\mathrm{EOF}}-\frac{\left[u_{2}\right]-U_{\mathrm{EOF}}}{\tau_{F}} .}
\end{aligned}
$$

As Eqs. (3) indicate, we calculate the frictional torque in the direction of the leading EOF using the lower-layer wind alone but split this torque across both layers in different proportions depending on the values of $\tau_{\mathrm{BT}}$ and $\tau_{\mathrm{BC}}$. For barotropic friction $\left(\tau_{\mathrm{BC}} \rightarrow \infty\right)$, the frictional torque is split equally between both layers so that no baroclinicity is generated. For baroclinic friction $\left(\tau_{\mathrm{BT}}=\infty\right)$, we apply equal and opposite torques in both layers so that there is no net force on the column. In the control setting $\left(\tau_{\mathrm{BC}}=\tau_{\mathrm{BT}}\right)$, friction has both a baroclinic and a barotropic effect. Note that this formulation produces a negative damping rate on the right-hand side of the $\left[F_{1}\right]$ equation when $\tau_{\mathrm{BC}}<\tau_{\mathrm{BT}}$, which implies that the dissipative term is actually speeding up the upper-level flow. However, it can be shown that the coupled system is stable because the upper-level torque is calculated using the lower-level wind rather than the upper-level wind. As shown below, there is no change in the dynamics as the system transitions across the $\tau_{\mathrm{BC}}=\tau_{\mathrm{BT}}$ threshold.

Figure 4 describes the sensitivity of the 20-day zonalindex memory (Fig. 4a), the maximum logarithmic decay rate (Fig. 4b) and the long-lag logarithmic decay rate (Fig. 4c) of the zonal-index autocorrelation, and the long-lag eddy feedback (Fig. 4d) when the frictional time scale is changed for the zonal-mean flow (green), the zonal-mean flow in the direction of the leading EOF alone (magenta), or its barotropic (blue) or baroclinic (red) components in that direction. As already shown above, changing the mean friction and the EOF friction produces similar results in all cases. We can also see that zonal-index memory is very sensitive to barotropic friction, increasing with decreasing friction, while the 

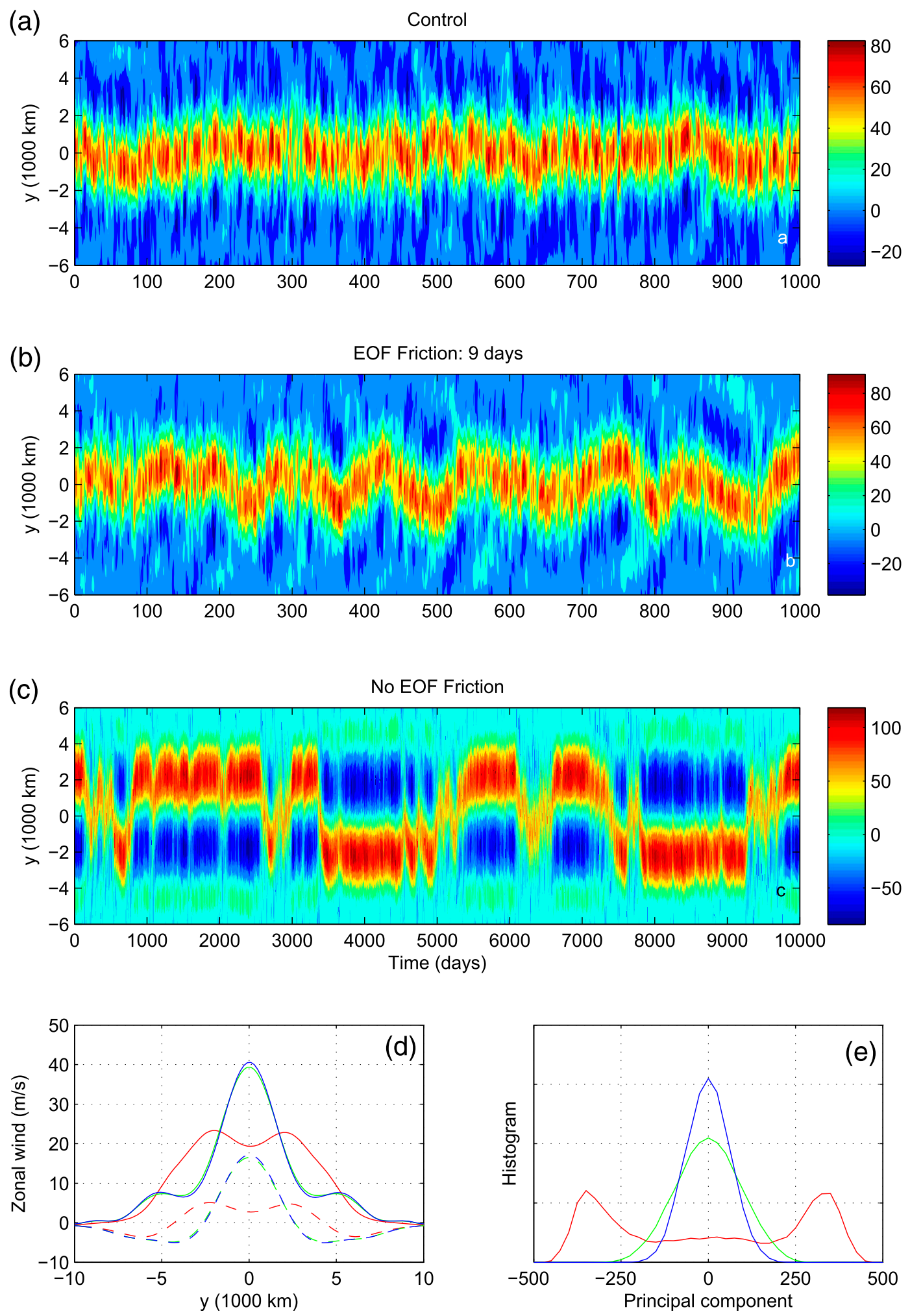

FIG. 3. (a) Sample time series of barotropic zonal-mean zonal wind $\left(\mathrm{m} \mathrm{s}^{-1}\right)$ for the control simulation. (b) As in (a), but for a simulation with reduced EOF friction. (c) As in (a), but for a simulation with no EOF friction (note the different horizontal scale). (d) Climatological zonal-mean zonal wind (lower layer dashed) for the same simulations in (a) (blue), (b) (green), and (c) (red). (e) Histograms of the principal components for the leading EOF in the same simulations. 
a) 20-day autocorrelation

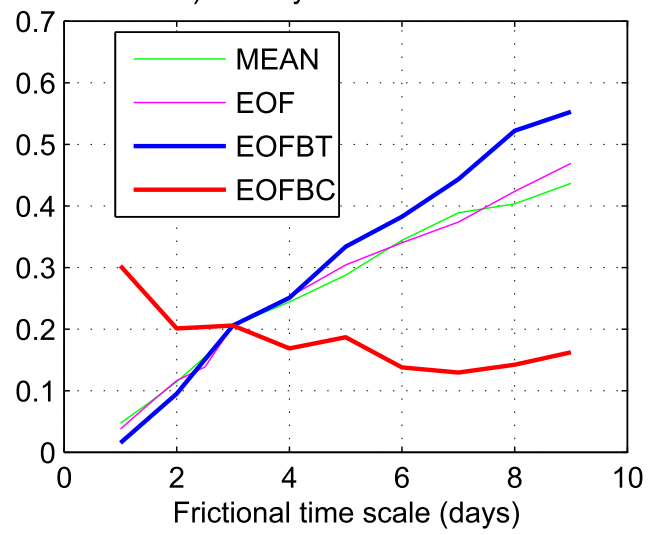

c) Long-lag log decay rate (days ${ }^{-1}$ )

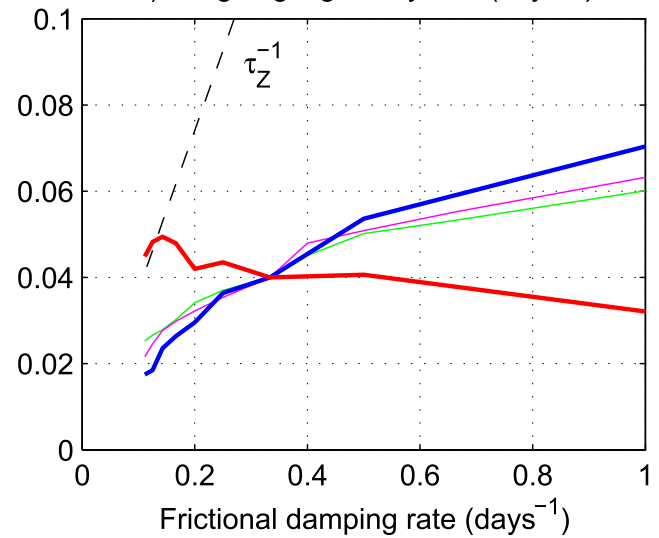

b) Maximum log decay rate (days ${ }^{-1}$ )

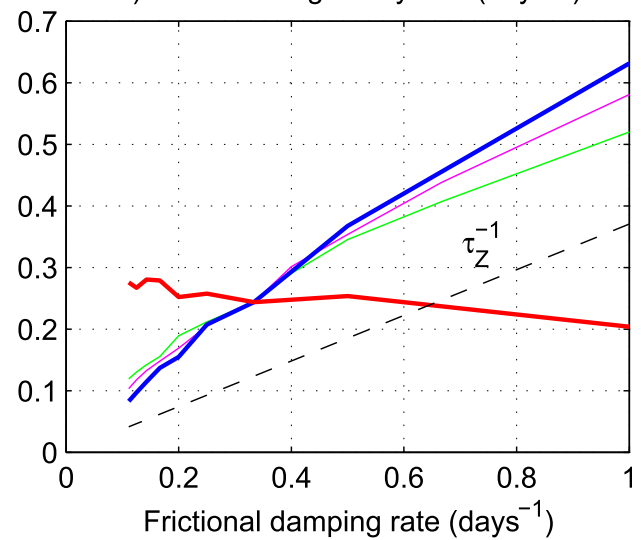

d) Eddy feedback coefficient (days ${ }^{-1}$ )

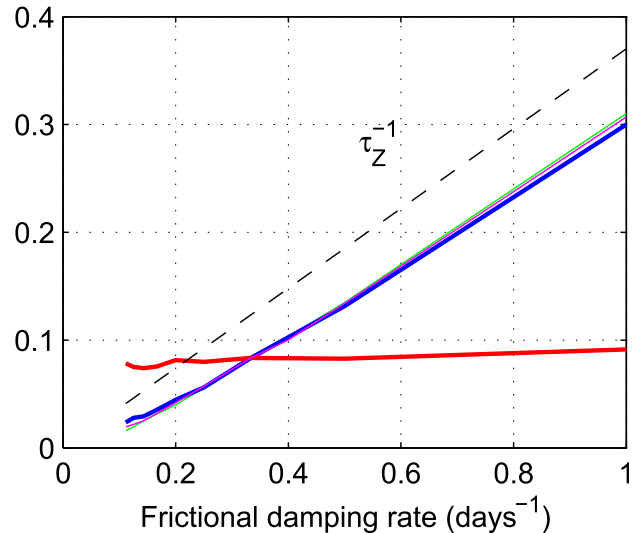

FIG. 4. (a) Sensitivity of the 20-day zonal-index autocorrelation to changes in the mean (green), EOF (magenta), barotropic (blue), and baroclinic (red) frictional time scale. (b) As in (a), but for its maximum logarithmic decay rate. (c) As in (a), but for the long-lag logarithmic decay rate. (d) As in (a), but for the inferred eddy feedback. The black dashed line in (b)-(d) shows the frictional damping of the zonal index $\tau_{Z}^{-1}$, where $\tau_{Z}=\alpha \tau_{F}$ is estimated using a constant $\alpha=2.7$.

sensitivity to baroclinic friction is much weaker and has the opposite sign (Fig. 4a). The strong sensitivity to barotropic friction is largely associated with changes in the short-lag decay, with the maximum zonal-index autocorrelation decay rate increasing strongly with increasing friction (Fig. 4b). In contrast, the long-lag logarithmic decay rate (estimated using the low-frequency forcing as discussed in section 2) also increases with barotropic friction (Fig. 4c), but these changes are much smaller than the changes in the frictional damping rate. As a result, the implied long-lag eddy feedback increases with barotropic friction, almost linearly (Fig. 4d).

Although the positive eddy feedback increases with baroclinic friction as expected from the arguments of Robinson (2000), this is a modest effect that cannot explain the sensitivity of the feedback to friction. Strikingly, Fig. 4d shows that the positive feedback is most sensitive to barotropic rather than baroclinic friction. The explanation is actually quite simple. As noted by Lorenz and Hartmann (2001), the low-frequency eddy momentum flux is very nearly balanced by friction so that Eq. (1) can be approximated in spectral space as $M \approx Z / \tau_{\mathrm{BT}}$ for $\omega \ll \tau_{\mathrm{BT}}^{-1}$. Thus, the low-frequency eddy momentum flux will scale as $m \sim z / \tau_{\mathrm{BT}}$ and defining an eddy feedback coefficient as $b \equiv m / z$, this coefficient will scale as $\tau_{\mathrm{BT}}^{-1}$ as shown in Fig. $4 \mathrm{~d}$. The logarithmic decay rate is a small residue between two terms that very nearly balance. We elaborate on these ideas in the next section.

\section{Discussion}

Figure 5a shows the eddy momentum forcing spectra $p_{m}$ for the control simulation and for the simulations with a $\tau_{\mathrm{EOF}}$ of 1 and 9 days. These spectra were calculated averaging 1000 different realizations over segments 500 days long. As friction decreases, the high-frequency 

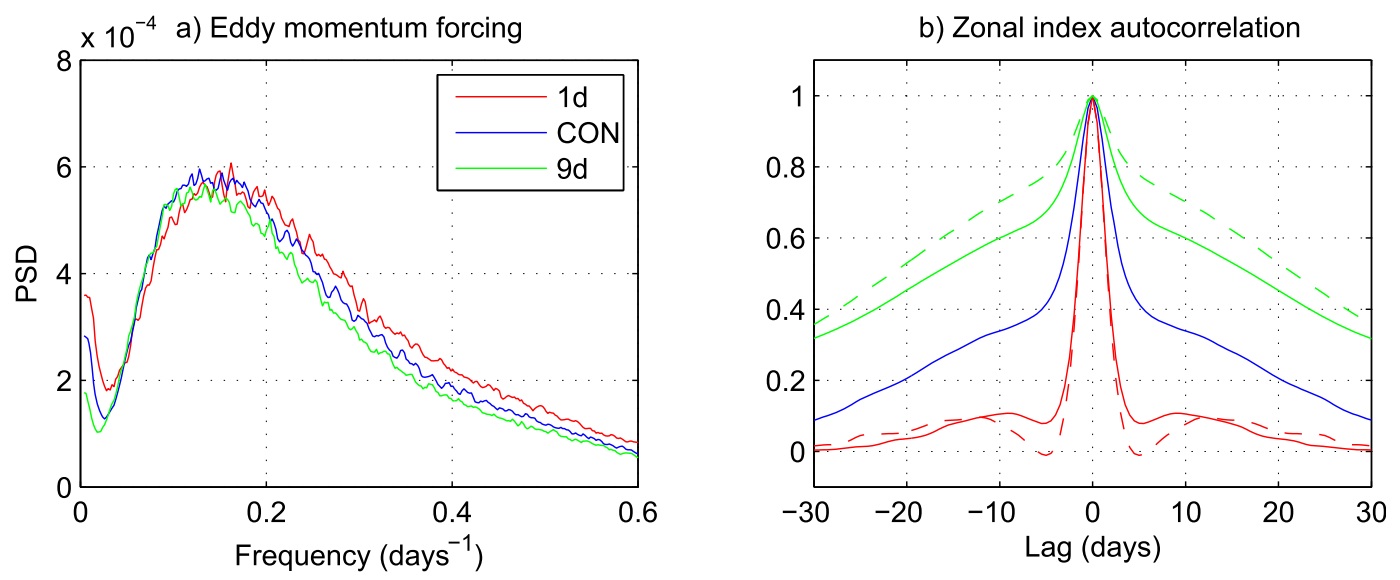

FIG. 5. (a) Eddy momentum forcing power spectra for the simulations with EOF friction with time scales of 1 (red), 3 [control (CON); blue], and 9 days (green). (b) Zonal-index autocorrelations for the same simulations (solid) and predicted by the offline model forced with the control eddy momentum forcing (dashed) for all time scales.

tail of the spectrum narrows and the low-frequency peak weakens relative to the high-frequency peak. Yet despite these systematic differences, the spectra are remarkably similar at the face of the profound differences in the strength of the eddy feedback noted above for these simulations.

In fact, one can understand the basic features of the sensitivity to friction by assuming that $m$ does not change with friction. This is illustrated by Fig. 5b, which compares the zonal-index autocorrelation in these simulations with the offline model predictions varying the frictional time scale but using the control eddy momentum forcing in all cases (if the actual eddy momentum forcing is used for each case, then the offline model gives a very good fit to the simulated autocorrelation; not shown). Although the agreement is far from perfect, it is striking how well we can do assuming that the eddy momentum forcing does not change.

The crucial factor that needs to be taken into account to understand the sensitivity of zonal-index persistence to friction is the spectral structure of the eddy forcing. To see this, it is useful to consider the relation between the power spectra for $z$ and $m$ :

$$
p_{z}(\omega)=\frac{p_{m}(\omega)}{\omega^{2}+\tau_{Z}^{-2}}
$$

where the zonal-index damping $\tau_{Z}=\alpha \tau_{\mathrm{BT}}$ is a function of barotropic friction alone. While in the simplest case of white eddy forcing $\left(p_{m}=\right.$ constant $), c_{z z}=\exp \left(-|\tau| / \tau_{Z}\right)$, and the logarithmic decay rate of the zonal-index autocorrelation scales as friction, the sensitivity of zonalindex persistence in our model is very different because $p_{m}$ has some structure. As shown in Fig. 5 and discussed in section $2, p_{m}$ displays a sharp low-frequency peak and a much broader peak at synoptic frequencies. The observed Southern Hemisphere eddy forcing spectrum has a similar structure (Lorenz and Hartmann 2001).

Consider now the case in which $p_{m}$ has a peak with width $\delta_{\omega}$ centered at zero frequency [i.e., $p_{m}(\omega)$ goes to zero for $\left.|\omega|>\delta_{\omega}\right]$. When this peak is sharp $\left(\delta_{\omega} \ll \tau_{Z}^{-1}\right)$, we can approximate

$$
p_{z}(\omega) \approx \tau_{Z}^{2} p_{m}(\omega)
$$

In that limit the spectral structure of $p_{z}(\omega)$ is equal to that of $p_{m}(\omega)$ and independent of friction, and the same is true for its inverse Fourier transform, the zonal-index autocovariance $C_{z z}(\tau)$. Thus, friction only affects the variance of $z$ and the amplitude of $C_{z z}$, but not $c_{z z}$. Since the logarithmic decay rate of $c_{z z}$ is independent of $\tau_{Z}$, the implied "eddy feedback" must increase with friction even if the actual $m$ does not change.

More generally, if $p_{m}$ has a peak at near-zero frequencies, then the $\left(\omega^{2}+\tau_{Z}^{-2}\right)^{-1}$ factor in Eq. (4) will sharpen that peak and hence flatten $c_{z z}$. Ultimately, the sharpness of the $p_{z}$ peak and the logarithmic decay rate for $c_{z z}$ will be controlled by the lesser between $\delta_{\omega}$ and $\tau_{Z}^{-1}$. In the sharp-peak limit $\left(\delta_{\omega} \ll \tau_{Z}^{-1}\right)$, the autocorrelation decay rate is insensitive to friction and the eddy feedback increases linearly with $\tau_{Z}^{-1}$ as discussed above. In contrast, when the peak is broad $\left(\delta_{\omega} \gg \tau_{Z}^{-1}\right.$, white forcing limit) the zonal-index autocorrelation decays as friction and there is no eddy feedback. In our simulations, the low-frequency $p_{m}$ peak is quite sharp and the long-lag logarithmic decay rate of the zonal-index autocorrelation is only weakly sensitive to friction-this requires that the eddy feedback scales almost linearly with friction as noted above (cf. Figs. 4c,d). However, the peak is not so sharp that the autocorrelation 
(a)

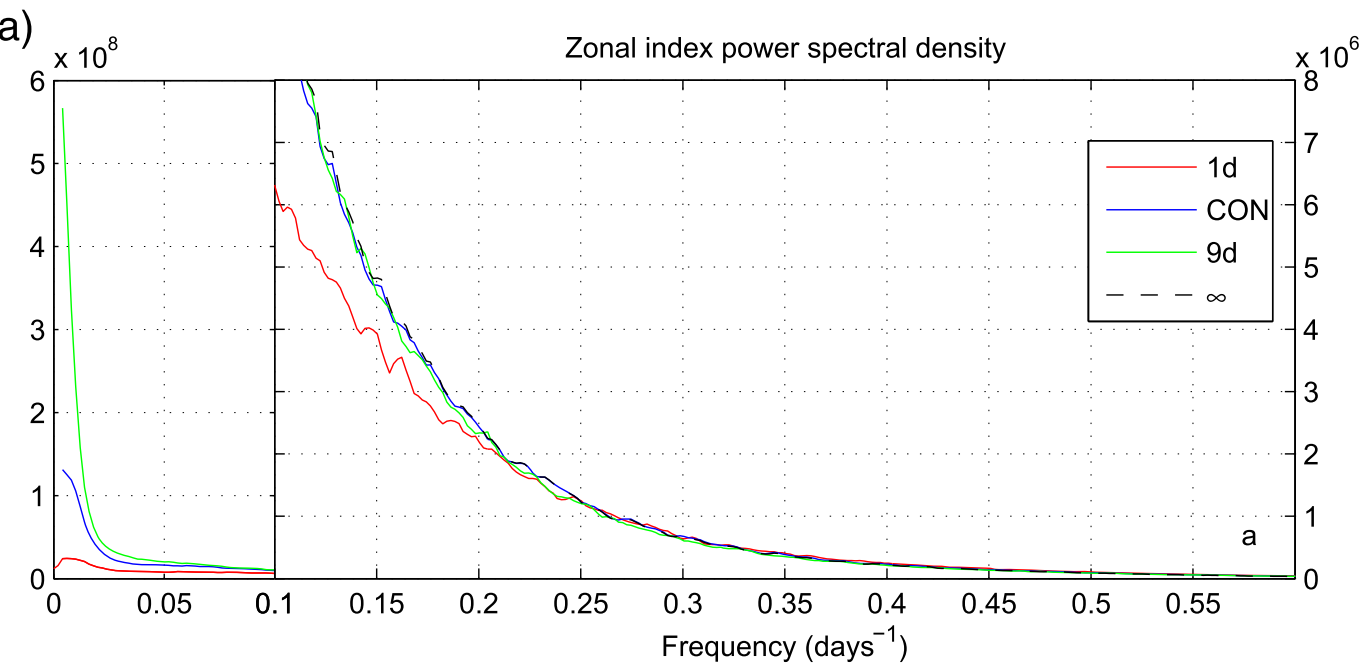

(b)

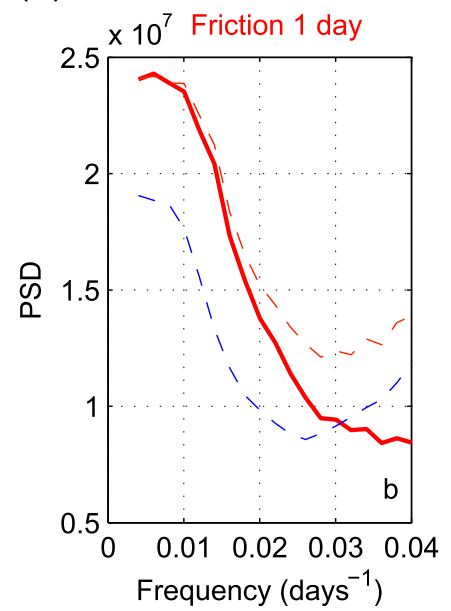

(c)

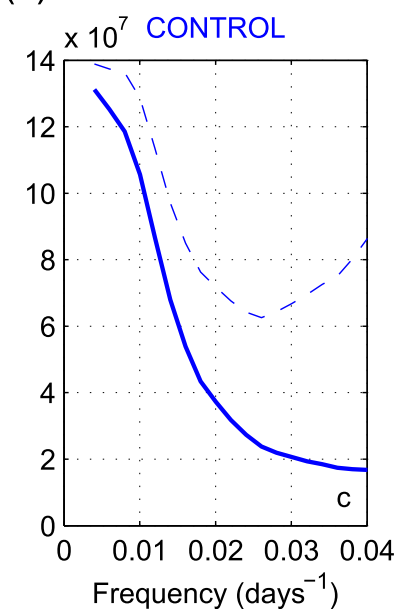

(d)

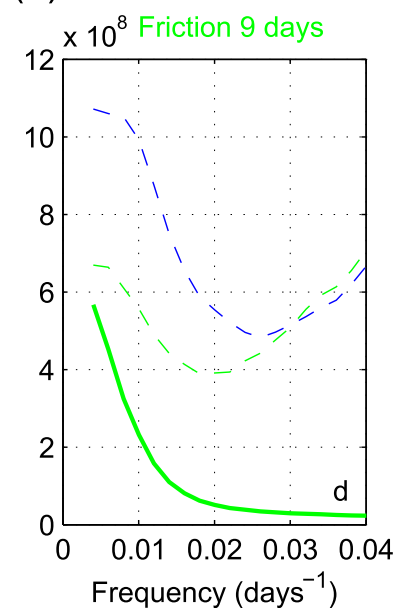

FIG. 6. (a) Zonal-index power spectra for the simulations with EOF friction with time scales of 1 (red), 3 (CON; blue), and 9 days (green), and the $\tau_{F}=\infty$ prediction (dashed black). Note the different ordinate for $\omega$ greater or less than $0.1 \mathrm{day}^{-1}$. (b) Zonal-index power spectrum $p_{z}$ (solid) for the simulation with 1-day friction, compared to $\tau_{Z}^{2} p_{m}$ (dashed) using the eddy forcing power spectrum for that run (dashed red) or the control eddy forcing power spectrum (dashed blue). (c),(d) As in (b), but for the control run and the run with 9-days friction, respectively.

logarithmic decay rate can be effectively regarded as constant.

While the above analysis can explain the long-lag behavior of the zonal-index autocorrelation, after the response to the high-frequency eddy forcing spectrum dies out, at shorter lags we also need to consider how that part of the response changes with friction. In the fast limit $\omega \gg \tau_{Z}^{-1}$, Eq. (4) may be approximated as

$$
p_{z}(\omega) \approx \frac{p_{m}(\omega)}{\omega^{2}} .
$$

Thus, for the high-frequency zonal-index response not only the structure of $p_{z}$ and $C_{z z}$ but also the variance of the response will be unchanged with varying friction when $m$ is fixed. Since, as discussed above, the amplitude of the low-frequency response scales as $\tau_{Z}^{2}$, the end result is that with stronger (weaker) friction, the highfrequency response will be more (less) dominant. This explains the more rapid short-lag decay in the zonalindex autocorrelation and the overall persistence drop with stronger friction.

We assess in Fig. 6 the relevance of these approximations for explaining the sensitivity of $p_{z}$ to friction in our model. Figure 6a shows the zonal-index power spectrum for the same three simulations of Fig. 5, using a different vertical scale for the low- and high-frequency parts of the spectrum. The latter is almost insensitive to friction and agrees very well with the $\tau_{Z} \rightarrow \infty$ prediction [Eq. (6)], shown with black dashed line. In contrast, the 
low-frequency peak sharpens and increases by over an order of magnitude when friction is reduced from 1 to 9 days.

Figures $6 \mathrm{~b}-\mathrm{d}$ zoom in on the low-frequency spectrum for these simulations. In all panels the thick solid line shows the zonal-index spectrum $p_{z}$ produced by the model in simulations with the frictional time scales indicated, while the dashed lines show the asymptotic prediction for $p_{z}$ in the sharp-peak limit $p_{z}=\tau_{Z}^{2} p_{m}$ [cf. Eq. (5)]. Two such predictions are shown, using either the $p_{m}$ spectrum for the corresponding simulation (same-color dashed line) or the control $p_{m}$ spectrum (blue dashed line), to assess the impact of changes in the eddy forcing. For these predictions, we also took into account the change in the vertical structure of the modes with friction ${ }^{1}$ to compute $\tau_{Z}=\alpha \tau_{F}$. Using the constant control value $\alpha=2.7$, the agreement is slightly worse.

With the strongest friction $\left(\tau_{F}=1\right.$ day; Fig. $\left.6 \mathrm{~b}\right)$, the simulated zonal-index spectrum agrees very well with the asymptotic prediction when $p_{m}$ from that simulation is used (dashed red line). There is no frictional sharpening of the spectrum, and $p_{m}$ and $p_{z}$ have the same spectral structure as predicted by the theory. When we use the control $p_{m}$ spectrum instead (dashed blue line), the gross spectral structure is still reproduced but the predicted $p_{z}$ peak is a bit too sharp and its amplitude is underestimated. This can explain the biases with fixed eddy forcing in Fig. $5 b$ for this value of friction: the zonal-index autocorrelation drops too steeply at short lags (because the low-frequency peak is too weak) and its long-lag logarithmic decay rate is too weak (because the peak is too sharp).

For the control value of friction ( $\tau_{F}=3$ days; Fig. 6c), the asymptotic prediction still works reasonably well even if the predicted $p_{z}$ peak is a bit too broad. Although the $p_{m}$ peak is much sharper than $\tau_{Z}^{-1}=(2.7 \times 3)^{-1} \approx 0.12$ days $^{-1}$, friction still produces some small additional sharpening of the spectrum. Finally, for $\tau_{F}=9$ days (Fig. 6d), the assumption $\delta_{\omega} \ll \tau_{Z}^{-1} \approx 0.04$ days $^{-1}$ required for the asymptotic limit [Eq. (5)] to hold is clearly not appropriate, but it is also not true that the peak width is negligible compared to the frictional damping rate (white forcing limit). In fact, Figs. $4 \mathrm{c}$ and $4 \mathrm{~d}$ show that the long-lag logarithmic decay rate of the zonal-index autocorrelation roughly equals half the frictional damping rate in that case, with the inferred eddy feedback accounting for the other half.

\footnotetext{
${ }^{1}$ From the structure of the regressed zonal-mean wind anomalies in both layers (see Z14 for details), we find that $\alpha$ changes from 2.5 to 3.0 when the frictional scale is reduced from 9 to 1 days.
}

\section{Concluding remarks}

In a very influential study, Lorenz and Hartmann (2001) noted that enhanced spectral power of the eddy momentum forcing at low frequencies increases the predictability of Southern Hemisphere jet variability. They attributed this enhanced power to the organization of the synoptic eddies by the anomalous mean flow and eloquently illustrated their argument by showing that a simple linear feedback model, in which the anomalous eddy forcing is modeled to be proportional to the meanflow anomaly, could reproduce quite well the observed low-frequency jet variability. The notion of a feedback coefficient is very appealing because it provides a simple device for quantifying the enhanced persistence using a reduced damping coefficient. A lot of subsequent work has aimed at elucidating the dynamics of this feedback and/or quantifying the feedback coefficient.

While the simple linear feedback model elegantly illustrates the importance of the synoptic eddy organization by the mean flow for the persistence of lowfrequency variability, the results presented here point to some shortcomings of the use of this model as a closure. A feedback coefficient is most meaningful when the ratio between the mean-flow and eddy forcing anomalies remains approximately constant as the mean flow changes, but this is not what happens in our model when friction is varied. As friction increases and the low-frequency mean-flow anomalies weaken, the low-frequency eddy momentum forcing anomalies do not weaken but the feedback coefficient increases instead. While it is possible to interpret this result in terms of a friction-dependent feedback coefficient, this is somewhat misleading in that it suggests that the eddies are adapting to compensate for the changes in friction even when the eddies do not change. A simpler interpretation is that the decay rate of the low-frequency mean-flow anomalies is insensitive to friction when this variability occurs in time scales much longer than the frictional damping rate.

It is also not clear that the dynamics of the eddy feedback can be characterized by a single time scale in our model, even with fixed friction. The fact that the zonal-index autocorrelation exhibits Gaussian rather than exponential decay at long lags (the autocorrelation decay rate increases linearly with lag; cf. Fig. 1c) suggests that the eddy feedback coefficient might be sensitive to the temporal scale of the anomalies. This is consistent with the spectral analysis of section 4 , which shows that the ratio between the low-frequency $m$ and $z$ anomalies depends on frequency except in the sharppeak limit $\delta_{\omega} \ll \tau_{Z}^{-1}$, where $\delta_{\omega}$ is the width of the spectral peak in the eddy momentum forcing. However, because in that limit the feedback coefficient simply scales as 
friction [cf. Eq. (5)], the decorrelation rate for the meanflow anomalies is defined by a small residual between two large quantities. Thus, in the limit in which the notion of a linear feedback is applicable-a limit possibly relevant for the atmosphere- the key parameter should be the long-lag decorrelation rate of the mean-flow anomalies rather than the feedback coefficient.

It is still an open question what determines this decorrelation rate or, equivalently, the width of the low-frequency spectral peak. The results presented here suggest that while friction has some effect on this parameter (cf. Figs. 4c, 5a), it does not define the parameter in any simple way. Analysis of the sensitivity to other external parameters in our model indicates that the same is true for the diabatic time scale (not shown). This suggests that the long-lag zonal-index autocorrelation decay rate is really defined by an internal dynamical time scale, consistent with Gerber and Vallis (2007). To construct a predictive model for this time scale, we need to better understand the dynamics of the coupled low-frequency barotropicbaroclinic variability described by Zurita-Gotor et al. (2014).

We conclude with a quick summary of the main results of our study:

- The sensitivity of zonal-index persistence to friction is dominated by the direct damping effect. One can understand to first order the changes in the zonalindex autocorrelation function with changing friction, assuming that the eddy forcing does not change.

- The main factor controlling this sensitivity is the structure of the eddy momentum forcing spectrum, which is very different from white noise. This spectrum consists of a broad peak at synoptic frequencies and a much sharper low-frequency peak.

- While the short-lag persistence depends on the relative amplitude of both peaks, the response to the synoptic peak dies out (because this peak is much broader) and the long-lag autocorrelation decay rate is only determined by the structure of the low-frequency peak.

- With increasing friction, zonal-index persistence drops at all lags because the high-frequency response becomes more dominant (friction damps the low-frequency variability preferentially). The decreased persistence is thus associated with faster initial decay of the autocorrelation function.

- Although the low-frequency zonal-index variance always decreases with friction, the temporal structure of this variability is weakly sensitive to friction when the low-frequency spectral peak is sharper than the frictional damping rate. In that limit, the long-lag autocorrelation decay rate changes little with friction, which requires the feedback coefficient to compensate for changes in friction.

Although the model used for this study is very idealized, the above-mentioned considerations do not depend on the specifics of the model used and we expect them also to be relevant for more realistic models, provided that the eddy momentum forcing spectrum exhibits a similar structure. The main caveat is that, on the sphere, friction also affects the latitude of the mean jet (Robinson 1997), which is one of the best-known factors affecting zonal-index persistence in comprehensive models (Kidston and Gerber 2010; Arakelian and Codron 2012), presumably because of differences in propagation at high and low latitudes (Barnes et al. 2010). Since this effect is absent in our model because of its meridional symmetry and use of a uniform beta, it is likely that changes in the mean state are more important for the sensitivity of zonal-index persistence to friction in the atmosphere than suggested by our study.

Acknowledgments. This work has benefited from inspiring discussions with Ed Gerber and the useful suggestions of two anonymous reviewers. Financial support from Projects DEVIAJE (Grant CGL2009-06948) and COMETH (Grant CGL2012-30641) from the Ministry of Science and Innovation of Spain is gratefully acknowledged.

\section{APPENDIX}

\section{Producing a Fixed Climatology with Varying Friction}

One confounding factor when analyzing the sensitivity to friction is that the mean state is also affected by changes in friction, which may make interpretation of the results ambiguous. Chen and Plumb (2009) devised a method to remove that effect by adding a constant torque that compensates the changes in the mean frictional force without affecting the variability. In particular, if the climatological lower-layer wind with the control value of friction $\tau_{F}$ is $\bar{U}_{c}$, then for a perturbed value of friction $\tau_{F}^{\prime}$ they model the frictional term as

$$
\left[\text { Fric }^{\mathrm{CP}}\right]=-\frac{\left[u_{2}\right]}{\tau_{F}^{\prime}}+\left(\frac{1}{\tau_{F}^{\prime}}-\frac{1}{\tau_{F}}\right) \bar{U}_{c} .
$$

Since the last term on the right-hand side is independent of time, the zonal wind is damped with time scale $\tau_{F}^{\prime}$. On the other hand, it is obvious that the mean state $\left[\bar{u}_{2}\right]=\bar{U}_{c}$ is an equilibrium solution of the system, since that would produce the same frictional torque as in the control run. 
However, it may not be the only equilibrium solution. We have found that this method works as required for small changes in $\tau_{F}^{-1}$, but for large changes the system evolves to a different solution because the prescribed torque is too strong and creates an anomalous jet that modifies how waves propagate. This is why we prefer to use the alternative forcing procedure discussed in the text, which is fully relaxational and does not suffer from the same problem:

$$
[\text { Fric }]=-\frac{U_{\mathrm{EOF}}}{\tau_{F}^{\prime}}-\frac{\left[u_{2}\right]-U_{\mathrm{EOF}}}{\tau_{F}},
$$

where $U_{\mathrm{EOF}}$ is the component of the zonal wind in the direction of the leading EOF. In this method, we apply the perturbed damping in that direction alone (at all times). This method works well because, empirically, the meridional structure of the EOF is slightly sensitive to the modified friction (though the EOF ranking may change when $\tau_{F}^{\prime}$ is sufficiently strong). It is clear that this method converges again to the control climatology, since, by the symmetry of the model, $\bar{U}_{\mathrm{EOF}}=0$ and the mean frictional term agrees again with the control run.

If we assume that the zonal wind variability is strongly dominated by the leading EOF-that is, $\left[u_{2}\right](y, t) \approx \bar{U}_{c}(y)+U_{\mathrm{EOF}}(y, t)$ - then both methods are equivalent, since Eq. (A1) simplifies to

$$
\begin{aligned}
{\left[\mathrm{Fric}^{\mathrm{CP}}\right] } & \approx-\frac{\bar{U}_{c}+U_{\mathrm{EOF}}}{\tau_{F}^{\prime}}+\left(\frac{1}{\tau_{F}^{\prime}}-\frac{1}{\tau_{F}}\right) \bar{U}_{c} \\
& =-\frac{U_{\mathrm{EOF}}}{\tau_{F}^{\prime}}-\frac{\bar{U}_{c}}{\tau_{F}} \approx-\frac{U_{\mathrm{EOF}}}{\tau_{F}^{\prime}}-\frac{\left[u_{2}\right]-U_{\mathrm{EOF}}}{\tau_{F}} .
\end{aligned}
$$

We have found that both methods produce similar results when they converge. An additional advantage of using different damping time scales for the different modes is that with this method it is also possible to change the barotropic and baroclinic friction independently as in section 3 .

\section{REFERENCES}

Arakelian, A., and F. Codron, 2012: Southern Hemisphere jet variability in the IPSL GCM at varying resolutions. J. Atmos. Sci., 69, 3788-3799, doi:10.1175/JAS-D-12-0119.1.

Barnes, E. A., and C. I. Garfinkel, 2012: Barotropic impacts of surface friction on eddy kinetic energy and momentum fluxes: An alternative to the barotropic governor. J. Atmos. Sci., 69, 3028-3039, doi:10.1175/JAS-D-11-0243.1.
— , and D. W. J. Thompson, 2014: Comparing the roles of barotropic versus baroclinic feedbacks in the atmosphere's response to mechanical forcing. J. Atmos. Sci., 71, 177-194, doi:10.1175/JAS-D-13-070.1.

- D. L. Hartmann, D. M. W. Frierson, and J. Kidston, 2010: Effect of latitude on the persistence of eddy-driven jets. Geophys. Res. Lett., 37, L11804, doi:10.1029/2010GL043199.

Blanco-Fuentes, J., and P. Zurita-Gotor, 2011: The driving of baroclinic anomalies at different time scales. Geophys. Res. Lett., 38, L23805, doi:10.1029/2011GL049785.

Chen, G., and P. Zurita-Gotor, 2008: The tropospheric jet response to prescribed zonal forcing in an idealized atmospheric model. J. Atmos. Sci., 65, 2254-2271, doi:10.1175/ 2007JAS2589.1.

— the persistence of the zonal-index in an idealized atmospheric model. J. Atmos. Sci., 66, 3707-3720, doi:10.1175/ 2009JAS3165.1.

- I. M. Held, and W. Robinson, 2007: Sensitivity of the jet latitude to friction. J. Atmos. Sci., 64, 2899-2915, doi:10.1175/ JAS3995.1.

Gerber, E., and G. Vallis, 2007: Eddy-zonal flow interactions and the persistence of the zonal index. J. Atmos. Sci., 64, 32963311, doi:10.1175/JAS4006.1.

James, I. N., 1987: Suppression of baroclinic instability in horizontally sheared flows. J. Atmos. Sci., 44, 3710-3720, doi:10.1175/ 1520-0469(1987)044<3710:SOBIIH >2.0.CO;2.

Kidston, J., and E. P. Gerber, 2010: Intermodel variability of the poleward shift of the austral jet stream in the CMIP3 integrations linked to biases in 20th century climatology. Geophys. Res. Lett., 37, L09708, doi:10.1029/2010GL042873.

Lorenz, D., 2014: Understanding midlatitude jet variability and change using Rossby wave chromatography: Wave-mean flow interaction. J. Atmos. Sci., in press.

— , and D. Hartmann, 2001: Eddy-zonal flow feedback in the Southern Hemisphere. J. Atmos. Sci., 58, 3312-3327, doi:10.1175/ 1520-0469(2001)058<3312:EZFFIT >2.0.CO;2.

Peixoto, J. P., and A. H. Oort, 1992: Physics of Climate. SpringerVerlag, $520 \mathrm{pp}$.

Robinson, W., 1997: Dissipation dependence of the jet latitude. J. Climate, 10, 176-182, doi:10.1175/1520-0442(1997)010<0176: DDOTJL $>2.0 . \mathrm{CO} ; 2$.

_ 2000: A baroclinic mechanism for the eddy feedback on the zonal index. J. Atmos. Sci., 57, 415-422, doi:10.1175/ 1520-0469(2000)057<0415:ABMFTE $>2.0$. CO;2.

Simpson, I. R., T. G. Shepherd, P. Hitchcock, and J. F. Scinocca, 2013: Southern annular mode dynamics in observations and models. Part II: Eddy feedbacks. J. Climate, 26, 5220-5241, doi:10.1175/JCLI-D-12-00495.1.

Zurita-Gotor, P., 2007: The relation between baroclinic adjustment and turbulent diffusion in the two-layer model. J. Atmos. Sci., 64, 1284-1300, doi:10.1175/JAS3886.1.

_ - J. Blanco-Fuentes, and E. P. Gerber, 2014: The impact of baroclinic eddy feedback on the persistence of jet variability in the two-layer model. J. Atmos. Sci., 71, 410-429, doi:10.1175/ JAS-D-13-0102.1. 
Copyright of Journal of the Atmospheric Sciences is the property of American Meteorological Society and its content may not be copied or emailed to multiple sites or posted to a listserv without the copyright holder's express written permission. However, users may print, download, or email articles for individual use. 